\title{
ON THE POLYNOMIAL MOMENT PROBLEM
}

\author{
F. PAKOVICH
}

\section{Introduction}

In this paper we treat the following "polynomial moment problem": for complex polynomials $P(z), Q(z)=\int q(z) \mathrm{d} z$ and distinct $a, b \in \mathbb{C}$ such that $P(a)=P(b), Q(a)=Q(b)$ to find conditions under which

$$
\int_{a}^{b} P^{i}(z) q(z) \mathrm{d} z=0
$$

for all integer non-negative $i$.

The polynomial moment problem was proposed in the series of papers of M. Briskin, J.-P. Francoise and Y. Yomdin [1]-[5] as an infinitesimal version of the center problem for the polynomial Abel equation in the complex domain in the frame of a programme concerning the classical Poincaré center-focus problem for the polynomial vector field on the plane. It was suggested that the following "composition condition" imposed on $P(z)$ and $Q(z)=\int q(z) \mathrm{d} z$ is necessary and sufficient for the pair $P(z), q(z)$ to satisfy $\left(^{*}\right)$ : there exist polynomials $\tilde{P}(z), \tilde{Q}(z), W(z)$ such that

$$
P(z)=\tilde{P}(W(z)), \quad Q(z)=\tilde{Q}(W(z)), \quad \text { and } \quad W(a)=W(b) .
$$

It is easy to see that the composition condition is sufficient: since after the change of variable $z \rightarrow W(z)$ the way of integration becomes closed, the sufficientness follows from the Cauchy theorem. The necessity of the composition condition in the case when $a, b$ are not critical points of $P(z)$ was proved by $\mathrm{C}$. Christopher in [6] (see also the paper of N. Roytvarf [12] for a similar result) and in some other special cases by M. Briskin, J.-P. Francoise and Y. Yomdin in the papers cited above.

Nevertheless, in general the composition conjecture fails to be true. Namely, in the paper [9] a class of counterexamples to the composition conjecture was constructed. These counterexamples exploit polynomials $P(z)$ which admit double decompositions: $P(z)=A(B(z))=C(D(z))$, where $A(z), B(z), C(z)$,

Received May 14, 2002.

2000 Mathematics Subject Classification. Primary: 30E99, 30C99, 30D05; Secondary: 34C99, 14H99.

Key words and phrases. Poincaré center-focus problem, polynomial Abel equation, polynomials, moments, compositions, functional equations. 
$D(z)$ are non-linear polynomials. If $P(z)$ is such a polynomial and, in addition, $B(a)=B(b), D(a)=D(b)$ then for any polynomial $Q(z)$ which can be represented as $Q(z)=E(B(z))+F(D(z))$ for some polynomials $E(z), F(z)$ condition (*) is satisfied with $q(z)=Q^{\prime}(z)$. On the other hand, it was shown in [9] that if $\operatorname{deg} B(z)$ and $\operatorname{deg} D(z)$ are coprime then condition $\left({ }^{* *}\right)$ is not satisfied already for $Q(z)=B(z)+D(z)$.

Note that double decompositions with $\operatorname{deg} A(z)=\operatorname{deg} D(z), \operatorname{deg} B(z)=$ $\operatorname{deg} C(z)$ and $\operatorname{deg} B(z), \operatorname{deg} D(z)$ coprime are described explicitly by Ritt's theory of factorization of polynomials. They are equivalent either to decompositions with $A(z)=z^{n} R^{m}(z), B(z)=z^{m}, C(z)=z^{m}, D(z)=z^{n} R\left(z^{m}\right)$ for a polynomial $R(z)$ and $\operatorname{GCD}(n, m)=1$ or to decompositions with $A(z)=T_{m}(z)$, $B(z)=T_{n}(z), C(z)=T_{n}(z), D(z)=T_{m}(z)$ for Chebyshev polynomials $T_{n}(z)$, $T_{m}(z)$ and $\operatorname{GCD}(n, m)=1$ (see [11], [13]).

The counterexamples above suggest to weaken the composition conjecture as follows: polynomials $P(z), q(z)$ satisfy condition $\left({ }^{*}\right)$ if and only if $\int q(z) \mathrm{d} z$ can be represented as a sum of polynomials $Q_{j}$ such that

$(* * *) \quad P(z)=\tilde{P}_{j}\left(W_{j}(z)\right), \quad Q_{j}(z)=\tilde{Q}_{j}\left(W_{j}(z)\right), \quad$ and $\quad W_{j}(a)=W_{j}(b)$

for some $\tilde{P}_{j}(z), \tilde{Q}_{j}(z), W_{j}(z) \in \mathbb{C}[z]$. For the case when $P(z)=T_{n}(z)$ this statement was verified in [10]. Moreover, it was shown that for $P(z)=T_{n}(z)$ the number of terms in the representation $\int q(z) \mathrm{d} z=\sum_{j} Q_{j}(z)$ can be reduced to two.

In this paper we give a solution of the polynomial moment problem in the case when $P(z)$ is indecomposable that is when $P(z)$ can not be represented as a composition $P(z)=P_{1}\left(P_{2}(z)\right)$ with non-linear polynomials $P_{1}(z), P_{2}(z)$. In this case conditions $(* *)$ and $(* * *)$ are equivalent and the composition conjecture reduces to the following statement.

Theorem 1. Let $P(z), Q(z)=\int q(z) \mathrm{d} z$ be complex polynomials and let $a, b$ be distinct complex numbers such that $P(a)=P(b), Q(a)=Q(b)$, and

$$
\int_{a}^{b} P^{i}(z) q(z) \mathrm{d} z=0
$$

for $i \geq 0$. Suppose that $P(z)$ is indecomposable. Then there exists a polynomial $\tilde{Q}(z)$ such that $Q(z)=\tilde{Q}(P(z))$.

We also examine the following condition which is stronger than $\left(^{*}\right)$ :

$$
\int_{a}^{b} P^{i}(z) Q^{j}(z) Q^{\prime}(z) \mathrm{d} z=0
$$

for $i \geq 0, j \geq 0$. If $\gamma$ is a curve which is the image of the segment $[a, b]$ in $\mathbb{C}^{2}$ under the map $z \rightarrow(P(z), Q(z))$ then this condition is equivalent to the condition that $\int_{\gamma} \omega=0$ for all global holomorphic 1 -forms $\omega$ in $\mathbb{C}^{2}$ ("the moment condition"). For an oriented simple closed curve $\delta$ of class $C^{2}$ in $\mathbb{C}^{2}$ the moment condition is necessary and sufficient to be a boundary of a bounded analytic variety $\Sigma$ 
in $\mathbb{C}^{2}$; it is a special case of the result of R. Harwey and B. Lawson [7]. The case when $\delta$ is an image of $S^{1}$ under the map $z \rightarrow(f(z), g(z))$, where $f(z), g(z)$ are functions analytic in an annulus containing $S^{1}$ was investigated earlier by J. Wermer [14]: in this case the moment condition is equivalent to the condition that there exists a finite Riemann surface $\Sigma$ with border $S^{1}$ such that $f(z), g(z)$ have an analytic extension to $\Sigma$.

Unlike condition $\left(^{*}\right)$ the more restrictive moment condition imposed on polynomials $P(z), Q(z)$ turns out to be equivalent to composition condition (**). We show that actually even a weaker condition is needed.

Theorem 2. Let $P(z), Q(z)$ be complex polynomials and let $a, b$ be distinct complex numbers such that $P(a)=P(b), Q(a)=Q(b)$, and

$$
\int_{a}^{b} P^{i}(z) Q^{j}(z) Q^{\prime}(z) \mathrm{d} z=0
$$

for $0 \leq i \leq \infty, 0 \leq j \leq d_{a}+d_{b}-2$, where $d_{a}$ (resp. $d_{b}$ ) is the multiplicity of the point a (resp. b) with respect to $P(z)$. Then there exist polynomials $\tilde{P}(z), \tilde{Q}(z)$, $W(z)$ such that $P(z)=\tilde{P}(W(z)), Q(z)=\tilde{Q}(W(z))$, and $W(a)=W(b)$.

Note that if $a, b$ are not critical points of $P(z)$ that is if $d_{a}=d_{b}=1$ then conditions of the theorem reduce to condition $\left(^{*}\right)$ and therefore Theorem 2 includes as a particular case the result of C. Christopher.

\section{Proofs}

2.1. Lemmata about branches of $Q\left(P^{-1}(z)\right)$. Let $P(z)$ and $Q(z)$ be rational functions and let $U \subset \mathbb{C}$ be a domain in which there exists a single-valued branch $p^{-1}(z)$ of the algebraic function $P^{-1}(z)$. Denote by $Q\left(P^{-1}(z)\right)$ the complete algebraic function obtained by the analytic continuation of the functional element $\left\{U, Q\left(p^{-1}(z)\right)\right\}$. Since the monodromy group $G\left(P^{-1}\right)$ of the algebraic function $P^{-1}(z)$ is transitive this definition does not depend of the choice of $p^{-1}(z)$. Denote by $d\left(Q\left(P^{-1}(z)\right)\right)$ the degree of the algebraic function $Q\left(P^{-1}(z)\right)$ that is the number of its branches.

Lemma 1. Let $P(z), Q(z)$ be rational functions. Then

$$
d\left(Q\left(P^{-1}(z)\right)\right)=\operatorname{deg} P(z) /[\mathbb{C}(z): \mathbb{C}(P, Q)] .
$$

Proof. Since any algebraic relation over $\mathbb{C}$ between $Q\left(p^{-1}(z)\right)$ and $z$ supplies an algebraic relation between $Q(z)$ and $P(z)$ and vice versa we see that $d\left(Q\left(P^{-1}(z)\right)\right)=[\mathbb{C}(P, Q): \mathbb{C}(P)]$. As $[\mathbb{C}(P, Q): \mathbb{C}(P)]=[\mathbb{C}(z): \mathbb{C}(P)] /$ $[\mathbb{C}(z): \quad \mathbb{C}(P, Q)]$ the lemma follows now from the observation that $[\mathbb{C}(z): \mathbb{C}(P)]=\operatorname{deg} P(z)$.

Recall that by Lüroth theorem each field $k$ such that $\mathbb{C} \subset k \subset \mathbb{C}(z)$ and $k \neq \mathbb{C}$ is of the form $k=\mathbb{C}(R), R \in \mathbb{C}(z) \backslash \mathbb{C}$. Therefore, the field $\mathbb{C}(P, Q)$ is a proper subfield of $\mathbb{C}(z)$ if and only if $P(z)=\tilde{P}(W(z)), Q(z)=\tilde{Q}(W(z))$ for some rational functions $\tilde{P}(z), \tilde{Q}(z), W(z)$ with $\operatorname{deg} W(z)>1$; in this case we 
say that $P(z)$ and $Q(z)$ have a common right divisor in the composition algebra. The Lemma 1 implies the following explicit criterion which is essentially due to Ritt [11] (cf. also [6], [12]).

Corollary 1. Let $P(z), Q(z)$ be rational functions. Then $P(z)$ and $Q(z)$ have a common right divisor in the composition algebra if and only if

$$
Q\left(p^{-1}(z)\right)=Q\left(\tilde{p}^{-1}(z)\right)
$$

for two different branches $p^{-1}(z), \tilde{p}^{-1}(z)$ of $P^{-1}(z)$.

Proof. Indeed, by Lemma 1 , the field $\mathbb{C}(P, Q)$ is a proper subfield of $\mathbb{C}(z)$ if and only if $d\left(Q\left(P^{-1}(z)\right)\right)<\operatorname{deg} P(z)$. On the other hand, the last inequality is clearly equivalent to condition (1).

Lemma 2. Let $P(z), Q(z)$ be rational functions, $\operatorname{deg} P(z)=n$. Suppose that there exist $a_{i} \in \mathbb{C}, 1 \leq i \leq n$, not all equal between themselves such that

$$
\sum_{i=1}^{n} a_{i} Q\left(p_{i}^{-1}(z)\right)=0 .
$$

If, in addition, the group $G\left(P^{-1}\right)$ is doubly transitive then $Q(z)=\tilde{Q}(P(z))$ for some rational function $\tilde{Q}(z)$.

Proof. Let $G \subset S_{n}$ be a permutation group and let $\rho_{G}: G \rightarrow G L\left(\mathbb{C}^{n}\right)$ be the permutation representation of $G$ that is $\rho_{G}(g), g \in G$ is the linear map which sends a vector $\vec{a}=\left(a_{1}, a_{2}, \ldots, a_{n}\right)$ to the vector $\overrightarrow{a_{g}}=\left(a_{g(1)}, a_{g(2)}, \ldots, a_{g(n)}\right)$. It is well known (see e.g. [15], Th. 29.9) that $G$ is doubly transitive if and only if $\rho_{G}$ is the sum of the identical representation and an absolutely irreducible representation. Clearly, the one-dimensional $\rho_{G}$-invariant subspace $E \subset \mathbb{C}^{n}$ corresponding to the identity representation is generated by the vector $(1,1, \ldots, 1)$. Therefore, since the Hermitian inner product $(\vec{a}, \vec{b})=a_{1} \bar{b}_{1}+a_{2} \bar{b}_{2}+\ldots+a_{n} \bar{b}_{n}$ is invariant with respect to $\rho_{G}$, the group $G$ is doubly transitive if and only if the subspace $E$ and its orthogonal complement $E^{\perp}$ are the only $\rho_{G}$-invariant subspaces of $\mathbb{C}^{n}$.

Suppose that (2) holds. In this case also

$$
\sum_{i=1}^{n} a_{i} Q\left(p_{\sigma(i)}^{-1}(z)\right)=0
$$

for all $\sigma \in G\left(P^{-1}\right)$ by the analytic continuation. To prove the lemma it is enough to show that $Q\left(p_{i}^{-1}(z)\right)=Q\left(p_{j}^{-1}(z)\right)$ for all $i, j, 1 \leq i, j \leq n$; then by Lemma 1 $[\mathbb{C}(z): \mathbb{C}(P, Q)]=\operatorname{deg} P(z)=[\mathbb{C}(z): \mathbb{C}(P)]$ and therefore $Q(z)=\tilde{Q}(P(z))$ for some rational function $\tilde{Q}(z)$. Assume the converse i.e. that there exists $z_{0} \in U$ such that not all $Q\left(p_{i}^{-1}\left(z_{0}\right)\right), 1 \leq i \leq n$, are equal between themselves. Without loss of generality we can suppose that all $Q\left(p_{i}^{-1}\left(z_{0}\right)\right), 1 \leq i \leq n$, are finite. Consider the subspace $V \subset \mathbb{C}^{n}$ generated by the vectors $\vec{v}_{\sigma}, \sigma \in G\left(P^{-1}\right)$, 
where $\vec{v}_{\sigma}=\left(Q\left(p_{\sigma(1)}^{-1}\left(z_{0}\right)\right), Q\left(p_{\sigma(2)}^{-1}\left(z_{0}\right)\right), \ldots, Q\left(p_{\sigma(n)}^{-1}\left(z_{0}\right)\right)\right.$. Clearly, $V$ is $\rho_{G\left(P^{-1}\right)^{-}}$ invariant and $V \neq E$. Moreover, it follows from (3) that $V$ is contained in the orthogonal complement $A^{\perp}$ of the subspace $A \subset \mathbb{C}^{n}$ generated by the vector $\left(\bar{a}_{1}, \bar{a}_{2}, \ldots, \bar{a}_{n}\right)$. Since $A \neq E$ we see that $V$ is a proper $\rho_{G}$-invariant subspace of $\mathbb{C}^{n}$ distinct from $E$ and $E^{\perp}$ that contradicts the assumption that the group $G\left(P^{-1}\right)$ is doubly transitive.

2.2. Lemma about preimages of domains. For a polynomial $P(z)$ denote by $c(P)$ the set of finite critical values of $P(z)$.

Lemma 3. Let $P(z)$ be a polynomial and let $V \subset \mathbb{C P}^{1}$ be a simply connected domain containing infinity such that $c(P) \cap V=\emptyset$. Then $P^{-1}\{V\}$ is conformally equivalent to the unit disk and $P^{-1}\{\partial V\}$ is connected.

Proof. Indeed, by the Riemann theorem $V$ is conformally equivalent to the unit disk $\mathbb{D}$ whenever $\partial V$ contains more than one point. It follows from $c(P) \cap V=\emptyset$ that $\partial V$ contains a unique point if and only if $P(z)$ has a unique finite critical value $c$ and $\partial V=c$; in this case there exist linear functions $\sigma_{1}, \sigma_{2}$ such that $\sigma_{1}\left(P\left(\sigma_{2}(z)\right)\right)=z^{n}, n \in \mathbb{N}$ and the lemma is obvious. Therefore, we can suppose that $V \cong \mathbb{D}$. Since $c(P) \cap V=\emptyset$ the restriction of the map $P(z): \mathbb{C P}^{1} \rightarrow \mathbb{C P}^{1}$ on $P^{-1}\{V\} \backslash P^{-1}\{\infty\}$ is a covering map. As $V \backslash \infty$ is conformally equivalent to the punctured unit disc $\mathbb{D}^{*}$ it follows from covering spaces theory that $P^{-1}\{V\} \backslash$ $P^{-1}\{\infty\}$ is a disjoint union of domains $\cup V_{i}$ conformally equivalent to $\mathbb{D}^{*}$ such that all induced maps $f_{i}: \mathbb{D}^{*} \rightarrow \mathbb{D}^{*}$ are of the form $z \rightarrow z^{l_{i}}, l_{i} \in \mathbb{N}$. But, as $P^{-1}\{\infty\}=\{\infty\}$, there may be only one such a domain. Therefore, the preimage $P^{-1}\{V\}$ is conformally equivalent to the unit disk. In particular, since $P^{-1}\{\partial V\}=\partial P^{-1}\{V\}$ we see that $P^{-1}\{\partial V\}$ is connected.

2.3. Proof of Theorem 2: the case of a regular value. In this section we investigate the case when $t_{0}=P(a)=P(b)$ is not a critical value of the polynomial $P(z)$. For a simple closed curve $M \subset \mathbb{C}$ denote by $D_{M}^{+}$(resp. by $D_{M}^{-}$) the domain that is interior (resp. exterior) with respect to $M$.

Let $L \subset \mathbb{C}$ be a simple closed curve such that $t_{0} \in L$ and $c(P) \subset D_{L}^{+}$. Denote by $\vec{L}$ the same curve considered as an oriented graph embedded into the complex plane. By definition, the graph $\vec{L}$ has one vertex $t_{0}$ and one counter-clockwise oriented edge $l$. Let $\vec{\Omega}=P^{-1}\{\vec{L}\}$ be an oriented graph which is the preimage of the graph $\vec{L}$ under the mapping $P(z): \mathbb{C} \rightarrow \mathbb{C}$, i.e. vertices of $\vec{\Omega}$ are preimages of $t_{0}$ and oriented edges of $\vec{\Omega}$ are preimages of $l$. As $L \cap c(P)=\emptyset$ the graph $\vec{\Omega}$ has $n=\operatorname{deg} P(z)$ vertices and $n$ edges. Furthermore, by Lemma 3 the graph $\vec{\Omega}=P^{-1}\left\{\partial D_{L}^{-}\right\}$is connected. Therefore, as a point set in $\mathbb{C}$ the graph $\vec{\Omega}$ is a simple closed curve. Let $l_{j}, 1 \leq j \leq n$, be oriented edges of $\vec{\Omega}$ and let $a_{j}$ (resp. $b_{j}$ ) be the starting (resp. ending) point of $l_{j}$. We will suppose that edges of $\vec{\Omega}$ are numerated by such a way that $a_{1}=a$ and that under a moving around the domain $P^{-1}\left\{D_{L}^{-}\right\}$along its boundary $\vec{\Omega}$ the edge $l_{i}, 1 \leq i \leq n-1$, is followed by the edge $l_{i+1}$ (see fig. 1 ). 

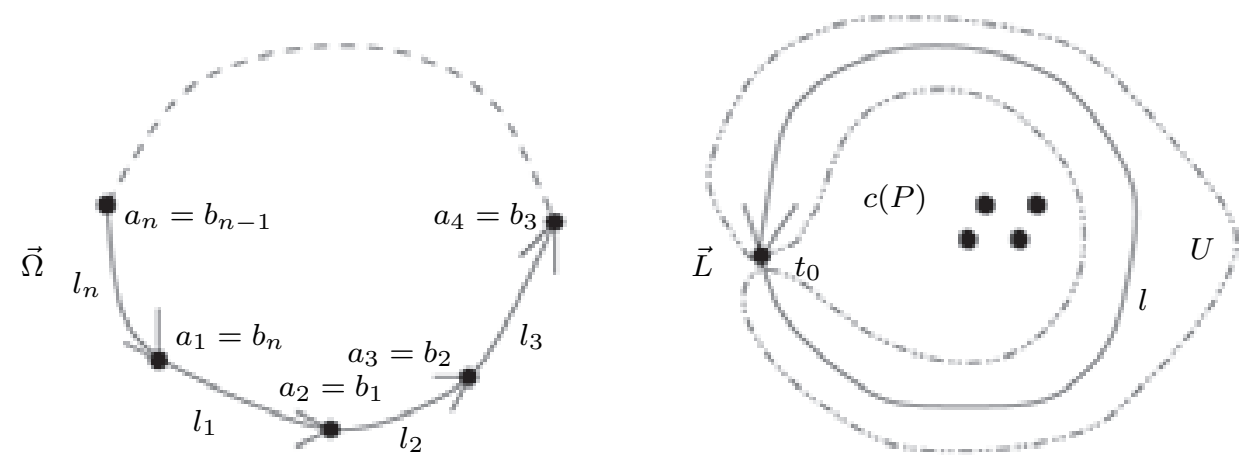

Figure 1

Let $U \subset \mathbb{C}$ be a simply connected domain such that $U \cap c(P)=\emptyset$ and $L \backslash\left\{t_{0}\right\} \subset U$. By the monodromy theorem, in such a domain there exist $n$ single-valued branches of $P^{-1}(t)$. Denote by $p_{j}^{-1}(t), 1 \leq j \leq n$, the single-valued branch of $P^{-1}(t)$ defined in $U$ by the condition $p_{j}^{-1}\left\{l \backslash t_{0}\right\}=l_{j} \backslash\left\{a_{j}, b_{j}\right\}$; such a numeration of branches of $P^{-1}(t)$ means that the analytic continuation of the functional element $\left\{U, p_{j}^{-1}(t)\right\}, 1 \leq j \leq n-1$, along $L$ is the functional element $\left\{U, p_{j+1}^{-1}(t)\right\}$. Let $l_{k}, k<n$, be the edge of $\Omega$ such that $b_{k}=b$ and let $\Gamma=\left\{l_{1}, l_{2}, \ldots, l_{k}\right\}$ be the oriented path in the graph $\Omega$ joining the vertices $a_{1}=a$ to $b_{k}=b$. For $t \in U$ set $\varphi(t)=\sum_{j=1}^{k} Q\left(p_{j}^{-1}(t)\right)$.

Consider an analytic function on $\mathbb{C P}^{1} \backslash L$

$$
I(\lambda)=\oint_{L} \frac{\varphi(t)}{t-\lambda} \mathrm{d} t=\int_{\Gamma} \frac{Q(z) P^{\prime}(z) \mathrm{d} z}{P(z)-\lambda} .
$$

More precisely, the integral above defines two analytic functions: one of them $I^{+}(\lambda)$ is analytic in $D_{L}^{+}$and the other one $I^{-}(\lambda)$ is analytic in $D_{L}^{-}$. Furthermore, calculating the Taylor expansion of $I^{-}(\lambda)$ at infinity and using integration by part we see that condition $\left(^{*}\right)$ reduces to the condition that $I^{-}(\lambda) \equiv 0$ in $D_{L}^{-}$. By a well-known result about integrals of the Cauchy type (see e.g. [8]) the last condition implies that $\varphi(t)$ is the boundary value on $L$ of the analytic function $I^{+}(\lambda)$ in $D_{L}^{+}$. It follows from the uniqueness theorem for boundary values of analytic functions that the functional element $\{U, \varphi(t)\}$ can be analytically continued along any curve $M \subset D_{L}^{+}$. As $c(P) \subset D_{L}^{+}$this fact implies that $\{U, \varphi(t)\}$ can be analytically continued along any curve $M \subset \mathbb{C}$. Therefore, by the monodromy theorem, the element $\{U, \varphi(t)\}$ extends to a single-valued analytic function in the whole complex plane. In particular, the analytic continuation of $\{U, \varphi(t)\}$ along any closed curve coincides with $\{U, \varphi(t)\}$. On the other hand, by construction the analytic continuation of $\{U, \varphi(t)\}$ along the curve $L$ is $\left\{U, \varphi_{L}(t)\right\}$, where $\varphi_{L}(t)=\sum_{j=2}^{k+1} Q\left(p_{j}^{-1}(t)\right)$. It follows from $\varphi(t)=\varphi_{L}(t)$ that $Q\left(p_{1}^{-1}(t)\right)=Q\left(p_{k+1}^{-1}(t)\right)$ and by Corollary 1 we conclude that $P(z)$ and $Q(z)$ have a common right divisor in the composition algebra. 
As the field $\mathbb{C}(P, Q)$ is a proper subfield of $\mathbb{C}(z)$ and $P(z), Q(z)$ are polynomials it is easy to prove that $\mathbb{C}(P, Q)=\mathbb{C}(W)$ for some polynomial $W(z)$, $\operatorname{deg} W(z)>1$. It means that $P(z)=\tilde{P}(W(z)), Q(z)=\tilde{Q}(W(z))$ for some polynomials $\tilde{P}(z), \tilde{Q}(z)$ such that $\tilde{P}(z)$ and $\tilde{Q}(z)$ have no a common right divisor in the composition algebra. Let us show that $W(a)=W(b)$. Since $t_{0}$ is not a critical value of the polynomial $P(z)=\tilde{P}(W(z))$ the chain rule implies that $t_{0}$ is not a critical value of the polynomial $\tilde{P}(z)$. Therefore, if $W(a) \neq W(b)$ then after the change of variable $z \rightarrow W(z)$ in the same way as above we find that $\tilde{P}(z)=\bar{P}(U(z)), \tilde{Q}(z)=\bar{Q}(U(z))$ for some polynomials $\bar{P}(z), \bar{Q}(z), U(z)$ with $\operatorname{deg} U(z)>1$ that contradicts the fact that $\tilde{P}(z), \tilde{Q}(z)$ have no a common right divisor in the composition algebra. This completes the proof in the case when $z_{0}$ is not a critical value of $P(z)$.

2.4. Proof of Theorem 2: the case of a critical value. Assume now that $t_{0}=P(a)=P(b)$ is a critical value of $P(z)$. In this case let $L$ be a simple closed curve such that $t_{0} \in L$ and $c(P) \backslash t_{0} \subset D_{L}^{+}$. Consider again a graph $\vec{\Omega}=P^{-1}\{\vec{L}\}$. Since $P^{-1}\left\{D_{L}^{-}\right\}$is still conformally equivalent to the unit disk by Lemma 3 , we see that the graph $\vec{\Omega}$ topologically is the boundary of a disc although it is not a simple closed curve any more. Let $l_{j}, 1 \leq j \leq n$, be oriented edges of $\vec{\Omega}$ and let $a_{j}$ (resp. $b_{j}$ ) be the starting (resp. the ending) point of $l_{j}$. Let us fix again such a numeration of edges of $\vec{\Omega}$ that $a_{1}=a$ and that under a moving around the domain $P^{-1}\left\{D_{L}^{-}\right\}$along its boundary $\vec{\Omega}$ the edge $l_{i}, 1 \leq i \leq n-1$, is followed by the edge $l_{i+1}$. As above denote by $U$ a domain in $\mathbb{C}$ such that $U \cap c(P)=\emptyset, L \backslash\left\{t_{0}\right\} \subset U$ and let $p_{j}^{-1}(t), 1 \leq j \leq n$, be the single-valued branch of $P^{-1}(t)$ defined in $U$ by the condition $p_{j}^{-1}\left\{l \backslash t_{0}\right\}=l_{j} \backslash\left\{a_{j}, b_{j}\right\}$. If $k<n$ is a number such that $b_{k}=b$ then for the same reason as above the function $\varphi(t)=\sum_{j=1}^{k} Q\left(p_{j}^{-1}(t)\right)$ extends to an analytic function in $U \cup D_{L}^{+}$but this fact does not imply now that $\varphi(t)$ extends to an analytic function in the whole complex plane since $D_{L}^{+}$does not contain $t_{0} \in c(P)$. Nevertheless, if $V$ is a simply connected domain such that $U \subset V$ and $t_{0} \notin V$ then $\varphi(t)$ still extends to a single-valued analytic function in $V$. In particular, the analytic continuation of $\{U, \varphi(t)\}$ along any simple closed curve $M$ such that $t_{0} \subset D_{M}^{-}$coincides with $\{U, \varphi(t)\}$.

Let $t_{1} \in U$ be a point and let $M_{1}$ (resp. $M_{2}$ ) be a simple closed curve such that $t_{1} \in M_{1}, M_{1} \cap c(P)=\emptyset$ and $D_{M_{1}}^{+} \cap c(P)=t_{0}$ (resp. $t_{1} \in M_{2}$, $M_{2} \cap c(P)=\emptyset$ and $\left.D_{M_{2}}^{+} \cap c(P)=c(P) \backslash t_{0}\right)$. Define a permutation $\rho_{1} \in S_{n}$ (resp. $\rho_{2} \in S_{n}$ ) by the condition that the functional element $\left\{U, p_{\rho_{1}(j)}^{-1}(t)\right\}$ (resp. $\left.\left\{U, p_{\rho_{2}(j)}^{-1}(t)\right\}\right)$ is the result of the analytic continuation of the functional element $\left\{U, p_{j}^{-1}(t)\right\}, 1 \leq j \leq n$, from $t_{1}$ along the curve $M_{1}$ (resp. $M_{2}$ ). Having in mind the identification of the set of elements $\left\{U, p_{j}^{-1}(t)\right\}, 1 \leq j \leq n$, with the set of oriented edges of the graph $\vec{\Omega}$ the permutations $\rho_{1}, \rho_{2}$ can be described as follows: $\rho_{1}$ cyclically permutes the edges of $\vec{\Omega}$ around the vertices from which they go 

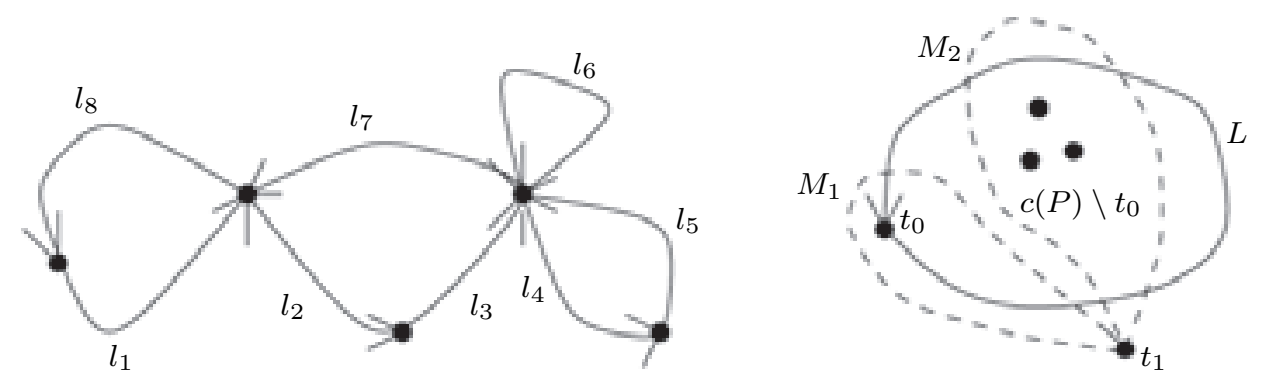

$\rho_{1}=(28)(467), \quad \rho_{2}=(18)(237)(45)$

FiguRe 2

while cycles $\left(j_{1}, j_{2}, \ldots, j_{k}\right)$ of $\rho_{2}$ correspond to simple cycles $\left(l_{j_{1}}, l_{j_{2}}, \ldots, l_{j_{k}}\right)$ of the graph $\vec{\Omega}$ and $\rho_{1} \rho_{2}=(12 \ldots n)$ (see fig. 2$)$.

To unload notation denote temporarily the element $\left\{U, Q\left(p_{i}^{-1}(t)\right)\right\}, 1 \leq i \leq n$, by $s_{i}$. Since $t_{0} \subset D_{M_{2}}^{-}$we have:

$$
0=\sum_{j=1}^{k} s_{\rho_{2}(j)}-\sum_{j=1}^{k} s_{j}=s_{\rho_{2}(k)}+\sum_{j=1}^{k-1}\left[s_{\rho_{2}(j)}-s_{j+1}\right]-s_{1} .
$$

Using $\rho_{1} \rho_{2}=(12 \ldots n)$ we can rewrite $(4)$ as

$$
s_{\rho_{1}^{-1}(k+1)}-s_{1}+\sum_{j=1}^{k-1}\left[s_{\rho_{2}(j)}-s_{\rho_{1} \rho_{2}(j)}\right]=0 .
$$

Therefore, by the analytic continuation

$$
s_{\rho_{1}^{f-1}(k+1)}-s_{\rho_{1}^{f}(1)}+\sum_{j=1}^{k-1}\left[s_{\rho_{1}^{f} \rho_{2}(j)}-s_{\rho_{1}^{f+1} \rho_{2}(j)}\right]=0
$$

for $f \geq 0$. Summing equalities (5) from $f=1$ to $f=o\left(\rho_{1}\right)$, where $o\left(\rho_{1}\right)$ is the order of the permutation $\rho_{1}$, changing the order of summing, and observing that

$$
\sum_{f=0}^{o\left(\rho_{1}\right)-1}\left[s_{\rho_{1}^{f} \rho_{2}(j)}-s_{\rho_{1}^{f+1} \rho_{2}(j)}\right]=s_{\rho_{2}(j)}-s_{\rho_{1}^{o\left(\rho_{1}\right)} \rho_{2}(j)}=0
$$

we conclude that

$$
\sum_{s=0}^{o\left(\rho_{1}\right)-1} Q\left(p_{\rho_{1}^{s}(k+1)}^{-1}(t)\right)=\sum_{s=0}^{o\left(\rho_{1}\right)-1} Q\left(p_{\rho_{1}^{s}(1)}^{-1}(t)\right)
$$

in $U$. Note that if $a, b$ are regular points of $P(z)$ then $\rho_{1}(1)=1, \rho_{1}(k+1)=k+1$ and $(6)$ reduces to the equality $Q\left(p_{k+1}^{-1}(t)\right)=Q\left(p_{1}^{-1}(t)\right)$. 
Since (6) holds for any polynomial $Q(z)$ such that $q(z)=Q^{\prime}(z)$ satisfies $\left(^{*}\right)$, substituting in $(6) Q^{j}(z), 2 \leq j \leq d_{a}+d_{b}-1$, instead of $Q(z)$ we see that

$$
\sum_{s=0}^{o\left(\rho_{1}\right)-1} Q^{j}\left(p_{\rho_{1}^{s}(k+1)}^{-1}(t)\right)=\sum_{s=0}^{o\left(\rho_{1}\right)-1} Q^{j}\left(p_{\rho_{1}^{s}(1)}^{-1}(t)\right)
$$

for all $j, 1 \leq j \leq \mathrm{d}_{b}+d_{b}-1$. Consider a Vandermonde determinant $D=$ $\left\|d_{j, i}\right\|$, where $d_{j, i}=Q^{j}\left(p_{i}^{-1}(t)\right), 0 \leq j \leq d_{a}+d_{b}-1$ and $i$ ranges the set of different indices from the cycles of $\rho_{1}$ containing 1 and $k+1$. Since (7) implies that $D=0$ we conclude again that $Q\left(p_{i}^{-1}(t)\right)=Q\left(p_{j}^{-1}(t)\right)$ for some $i \neq j$, $1 \leq i, j \leq n$. Therefore, $P(z)$ and $Q(z)$ have a common right divisor in the composition algebra and we can finish the proof by the same argument as in section 2.3 taking into account that the multiplicity of a point $c \in \mathbb{C}$ with respect to $P(z)=\tilde{P}(W(z))$ is greater or equal than the multiplicity of the point $W(c)$ with respect to $\tilde{P}(z)$.

2.5. Proof of Theorem 1. Suppose at first that $n=\operatorname{deg} P(z)$ is a prime number. In this case the degree of the algebraic function $Q\left(P^{-1}(t)\right)$ equals either $n$ or 1 since $d\left(Q\left(P^{-1}(t)\right)\right)$ divides $\operatorname{deg} P(z)$. If $d\left(Q\left(p^{-1}(t)\right)\right)=n$ then Puiseux expansions at infinity

$$
Q\left(p_{i}^{-1}(t)\right)=\sum_{k \leq k_{0}} a_{k} \varepsilon^{i k} t^{\frac{k}{n}}
$$

$1 \leq i \leq n, a_{k} \in \mathbb{C}, \varepsilon=\exp (2 \pi i / n)$, contain a coefficient $a_{k} \neq 0$ such that $k$ is not a multiple of $n$. Substituting (8) in the equality obtained by the analytic continuation of (6) along a curve going to the domain where series (8) converge, we conclude that $\varepsilon^{k}$ is a root of a polynomial with integer coefficients distinct from the $n$-th cyclotomic polynomial $\Phi_{n}(z)=1+z+\ldots+z^{n-1}$. Since $\varepsilon^{k}$ is a primitive $n$-th root of unity it is a contradiction. Therefore, $d\left(Q\left(p^{-1}(t)\right)\right)=1$ and $Q(z)=\tilde{Q}(P(z))$ for some polynomial $\tilde{Q}(z)$.

Suppose now that $n$ is composite. Since $P(z)$ is indecomposable the group $G\left(P^{-1}\right)$ is primitive by the Ritt theorem [11]. By the Schur theorem (see e.g. [15], Th. 25.3) a primitive permutation group of composite degree $n$ which contains an $n$-cycle is doubly transitive. Therefore, by Lemma 2 equality (6) implies that $Q(z)=\tilde{Q}(P(z))$ for some polynomial $\tilde{Q}(z)$.

\section{Acknowledgements}

I am grateful to Y. Yomdin for drawing my attention to the polynomial moment problem and for stimulating discussions.

\section{References}

[1] M. Briskin, J.-P. Francoise, Y. Yomdin, Une approche au probleme du centre-foyer de Poincaré, C. R. Acad. Sci., Paris, Sér. I Math. 326 (1998), 1295-1298.

[2] _ Center conditions, compositions of polynomials and moments on algebraic curves, Ergodic Theory Dyn. Syst. 19 (1999), 1201-1220. 
[3] Center conditions. II. Parametric and model center problems, Israel J. Math. 118 (2000), 61-82.

[4] Center conditions. III. Parametric and model center problems, Israel J. Math. 118 (2000), 83-108.

[5] - Generalized moments, center-focus conditions, and compositions of polynomials, Operator theory, system theory and related topics (Beer-Sheva/Rehovot, 1997), 161-185, Oper. Theory Adv. Appl., 123, Birkhäuser, Basel, 2001.

[6] C. Christopher, Abel equations: composition conjectures and the model problem, Bull. Lond. Math. Soc. 32 (2000), 332-338.

[7] F. Harvey, H. Lawson, On boundaries of complex analytic varieties. I, Ann. of Math. (2) 102 (1975), 223-290.

[8] N. Muskhelishvili, Singular integral equations, Boundary problems of function theory and their application to mathematical physics. P. Noordhoff N. V., Groningen, 1953.

[9] F. Pakovich, A counterexample to the "composition conjecture", Proc. Amer. Math. Soc. 130 (2002), 3747-3749.

[10] On polynomials orthogonal to all powers of a Chebyshev polynomial on a segment, preprint.

[11] J. Ritt, Prime and composite polynomials, Trans. Amer. Math. Soc. 23 (1922), 51-66.

[12] N. Roytvarf, Generalized moments, composition of polynomials and Bernstein classes, Entire functions in modern analysis (Tel-Aviv, 1997), 339-355, Israel Math. Conf. Proc., 15, Bar-Ilan Univ., Ramat Gan, 2001.

[13] A. Schinzel, Polynomials with special regard to reducibility, Encyclopedia of Mathematics and its Applications, 77. Cambridge University Press, Cambridge, 2000.

[14] J. Wermer, The hull of a curve in $C^{n}$, Ann. of Math. (2) 68 (1958), 550-561.

[15] H. Wielandt, Finite permutation groups, Academic Press, New York-London, 1964.

Max-Planck-Institut Für Mathematik, P.O. Box 7280, D-53072 Bonn, Germany.

E-mail address: pakovich@mpim-bonn.mpg.de 